\title{
MICROSTRUCTURE CHARACTERIZATION BY MEANS OF X-RAY MICRO-CT AND NANOINDENTATION MEASUREMENTS
}

\author{
MAGDALENA RAJCZAKOWSKA, DAMIAN STEFANIUK, DARIUSZ ŁYDŻBA \\ Wrocław University of Technology, Faculty of Civil Engineering, Institute of Geotechnics and Hydrotechnics, \\ Wybrzeże Wyspiańskiego 27, 50-370 Wrocław, Poland, \\ e-mail: magdalena.rajczakowska@pwr.edu.pl,damian.stefaniuk@pwr.edu.pl,dariusz.lydzba@pwr.edu.pl
}

\begin{abstract}
The aim of this paper is to present an example of the material microstructure characterization with the use of X-ray micro-CT and nanoindentation measurements. Firstly, the current scope of application of the aforementioned techniques is provided within different fields of science. Then, background of each of the methods is presented. The methodology of X-ray micro-CT is described with the emphasis on the Beer's law formulation. In addition, the basics of the nanoindentation technique are outlined and major formulas for the hardness and Young's modulus calculation are given. Finally, example results for a concrete sample are presented. The microstructure of the selected material is firstly characterized in terms of geometry using the results from the microtomograhy measurements, e.g., porosity and attenuation profiles, pore and aggregate size distribution, shape factor of pores, etc. Next, the results of the nanoindentation tests are provided, namely the hardness and Young's modulus versus the height of the sample. The influence of the number of tests and statistical analysis on the final results is underlined.
\end{abstract}

Key words: micro-CT, nanoindentation, microstructure, composite

\section{INTRODUCTION}

Modern material testing techniques have gained a significant role in understanding the processes occurring in the material microstructure. Scientists are able to expand their knowledge and use it for development of new materials or improvement of the existing ones. Next to the testing methods allowing observation of the microstructure's geometry, e.g., optical microscopy, scanning electron microscopy, X-ray micro-computed tomography, there are techniques providing the mechanical parameters of the microstructure components, e.g., the nanoindentation technique which is a modified hardness test applied to small volumes. This paper focuses on the application of X-ray micro-computed tomography and nanoindentation techniques for characterization of the materials. Both methods have been successfully implemented for characterization of various materials. Henry et al. [1] applied X-ray micro-CT for investigation of the heating effect on concrete's microstructure. Ren et al. [2] utilized the images from the micro-CT analysis to develop 2D meso-scale FE models to simulate crack propagation processes in concrete under uniaxial tension loading. There were also several attempts to study the porosity of concrete [3], ceramics [4] or soil [5]. Liu et al. [6] presented an imaging method to assess the homogeneity of asphalt concrete using X-ray computed tomography whereas Steppe et al. [7] applied computed microtomography for non-invasive determination of wood anatomical characteristics. On the other hand, nanoindentation technique has been commonly used to assess mechanical properties of concrete, cement paste or rocks [8]-[10]. It was also applied for measuring properties of biomaterials and tissues [11] and polymer nanocomposites in order to evaluate the surface mechanical properties down to the nanoscale level [12].

Nevertheless, there are few examples of combining the X-ray micro-computed Tomography and nanoindentation techniques in order to find complex information about the material microstructure, namely: its geometry and mechanical properties. Thus, the aim of this paper is to verify their usefulness as well as the effectiveness of this approach on an exemplary material.

The article is organized as follows. In the next section, the principles of X-ray micro-CT are presented. The construction of a typical scanner is shown, followed by the Beer's law formulation. Section 3 
introduces the theory of nanoindentation. Basic formulas for the measured parameters, e.g. material hardness and Young's modulus, are presented. The section is followed by sample results, where the exemplary outcome of the aforementioned measurements is provided and analyzed. Final conclusions end the paper.

\section{PRINCIPLES OF X-RAY MICRO-COMPUTED TOMOGRAPHY}

$\mathrm{X}$-ray micro-computed tomography is a nondestructive and noninvasive imaging method where individual projections recorded from different viewing directions are used to reconstruct the internal structure of the material. It provides an accurate map of the variation of X-ray absorption within the scanned object [13]. The construction a the typical scanner is presented in Fig. 1.

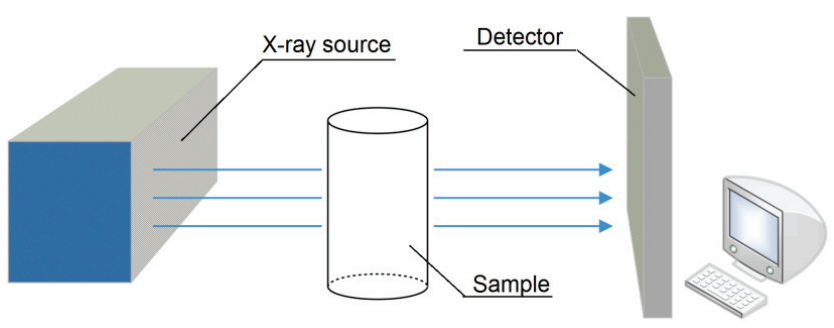

mathematical reconstruction process is necessary. Finally, the set of slices on the height of the object investigated is gathered, which allows for further image processing including binarization, etc. Based on the binary images, a variety of microstructure measures can be calculated, e.g., porosity, shape factor, specific surface, correlation functions, etc. In addition, a $3 \mathrm{D}$ reconstruction of the object's volume can be obtained and analyzed [14].

The total attenuation of an X-ray passing through the object can be expressed as a "line integral" - the sum of the absorptions along the path of the beam. The intensity $I$ of the transmitted X-ray beam, according to Beer's law, is verified by the relation [13]

$$
I(x)=I_{0} e^{-\mu x},
$$

where $I$ represents the intensity of the beam that have already passed through the sample, $I_{0}$ is the initial intensity of the beam (at $x=0$ ) and $\mu$ is the material's linear attenuation coefficient equal to

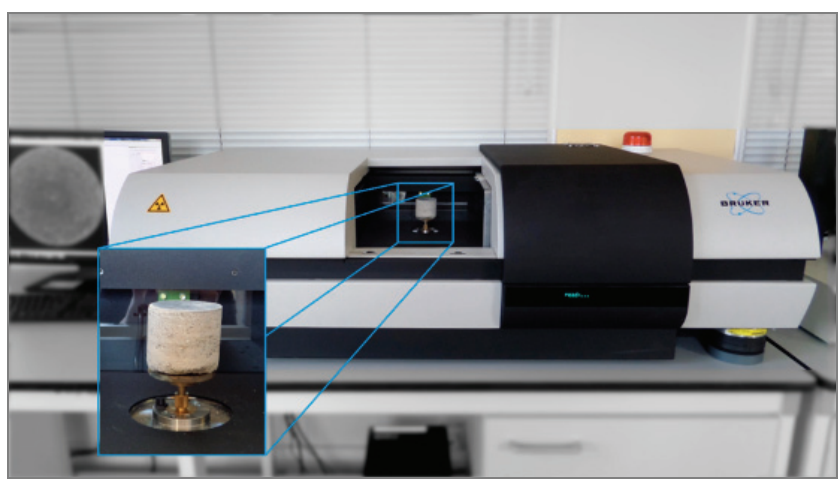

Fig. 1. The construction of the $\mu \mathrm{CT}$ system

The X-ray source is built of two electrodes: anode and cathode. Between them the high voltage is applied in order to produce radiation. The sample is rotating on the stage within the preset value of unit angle while the radiation is being transmitted. The projections, for each angle, are recorded on the detector covered by a scintillator The scintillator transforms the ionizing radiation (absorbed energy) into light. It is connected to a charge coupled device (CCD) camera that changes the light into the digital signal which is transferred to the computer. The scanning requires adjusting a large number of settings such as unit angle, use of filter, voltage, power of the X-ray source, etc. Analysis of each material needs a different set of parameters due to their unique properties. As a result of the scan, a collection of object projections is acquired. In order to obtain the cross-sections of the sample, the

$$
\mu=\sum f_{i} \mu_{i} \rho_{i}
$$

where $i$ denotes an atomic element, $\rho_{i}$ is the density of the material, $f_{i}$ is the atomic weight, $\mu_{i}$ represents the mass attenuation coefficient of the beam energy [13].

After differentiating (1) one obtains the following formula for the rate of attenuation with distance

$$
\frac{d I}{d x}=-\mu I .
$$

In the case of heterogeneous body, the attenuation coefficient $\mu$ varies with distance. Taking that into consideration, one can derive the following general formula [13]

$$
I(x)=I_{0} \exp \left(-\int \mu d x\right) .
$$




\section{THEORY OF NANOINDENTATION MEASUREMENTS}

Indentation is a commonly used technique to measure the mechanical parameters of the material. In the last 20 years it has been developed and nowadays it enables the measurements also in nano-scale. Nanoindentation method was developed by Doerner and Nix [15] and later by Oliver and Pharr [16]. It allows us to determine the hardness of the material investigated and its elastic modulus from depth sensing indentation load-displacement data (DSI). The method was successfully applied for characterization of mechanical properties of the materials, e.g., analysis of the effect of grain size and thickness on the thin film properties, development of nanocomposites, application of nanometer thick films for miniaturization of engineering and electronic composites, probing mechanical properties in small volumes. The principle of the nanoindentation method is based on pressing a hard diamond tip of known geometry (in this case, Berkovich triangular pyramid) into the sample (Fig. 2b). The load is increased to the preset value, depending on the type of material, etc. As a result of the test, load versus displacement curves are obtained as presented in Fig. 2a. Based on the aforementioned curve the following parameters are acquired: the maximum loading $P_{\max }$, the maximum displacement $h_{\max }$, the permanent depth of penetration $h_{f}$ and the elastic unloading stiffness $S=d P / d h$.

$$
H=\frac{P_{\max }}{A},
$$

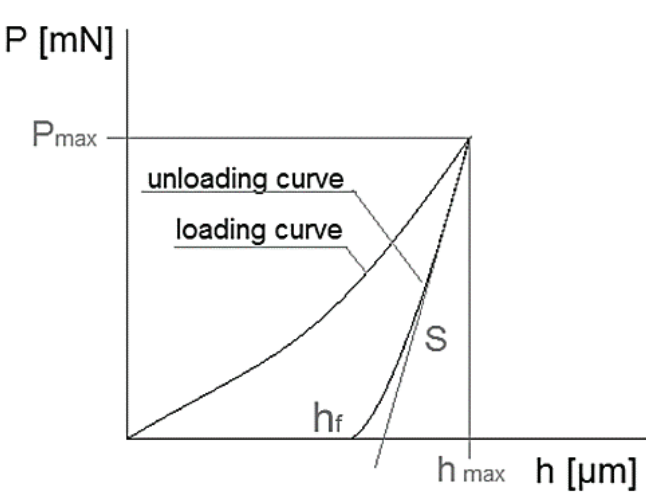

a)

$$
\begin{gathered}
S=\beta \frac{2}{\sqrt{\pi}} E_{\text {eff }} \sqrt{A} \\
\frac{1}{E_{\text {eff }}}=\frac{1-v^{2}}{E}+\frac{1-v_{i}^{2}}{E_{i}}
\end{gathered}
$$

where $A$ is a contact area between the indenter and the sample, $\beta$ is a dimensionless parameter taking into account the lack of axial symmetry of the indenter, $E_{\text {eff }}$ is the effective elastic modulus, $E$ is the elastic modulus of the material, $v$ is Poisson's ratio of the material, whereas $E_{i}$ and $v_{i}$ are the elastic parameters of the indentation tip. Knowing Poisson's ratio of the sample one can calculate Young's modulus $E$ of the material based on equation (7) [17].

\section{SAMPLE RESULTS}

The methods described in the previous sections were applied on a selected concrete specimen. Firstly, the sample was scanned with the use of SkyScan 1172 $\mathrm{X}$-ray. The X-ray tube voltage was set to $100 \mathrm{kV}$ and the tube's power was constant at $10 \mathrm{~W}$. The scan was performed using built-in $\mathrm{Al}+\mathrm{Cu}$ filter (Al $1 \mathrm{~mm}$ and $\mathrm{Cu} 0.05 \mathrm{~mm}$ ). In addition, for each scan the flat field correction was applied. The reconstruction was performed using NRecon based on Feldkamp algorithm. For the 3D visualization of the images, CTvox software is utilized.

In Fig. 3, the results of the material imaging are presented, namely the $3 \mathrm{D}$ reconstruction and the $2 \mathrm{D}$ images of the sample. Three components of the

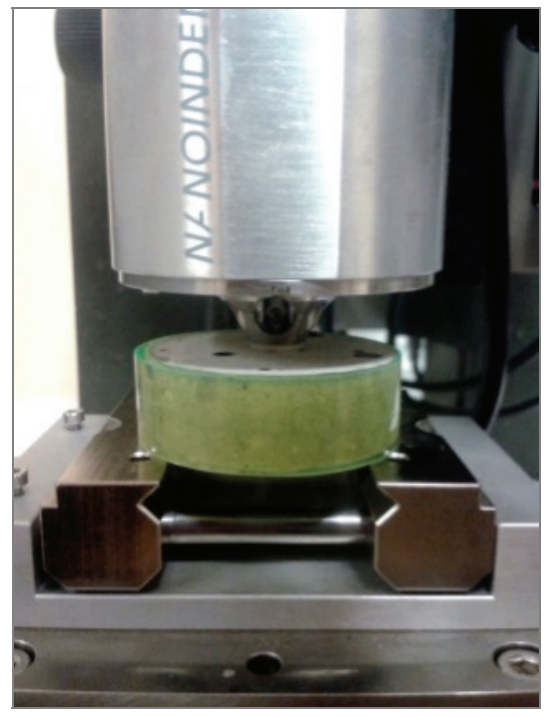

b)

Fig. 2. (a) Typical load-displacement curve [16]; (b) nanoindentation set 


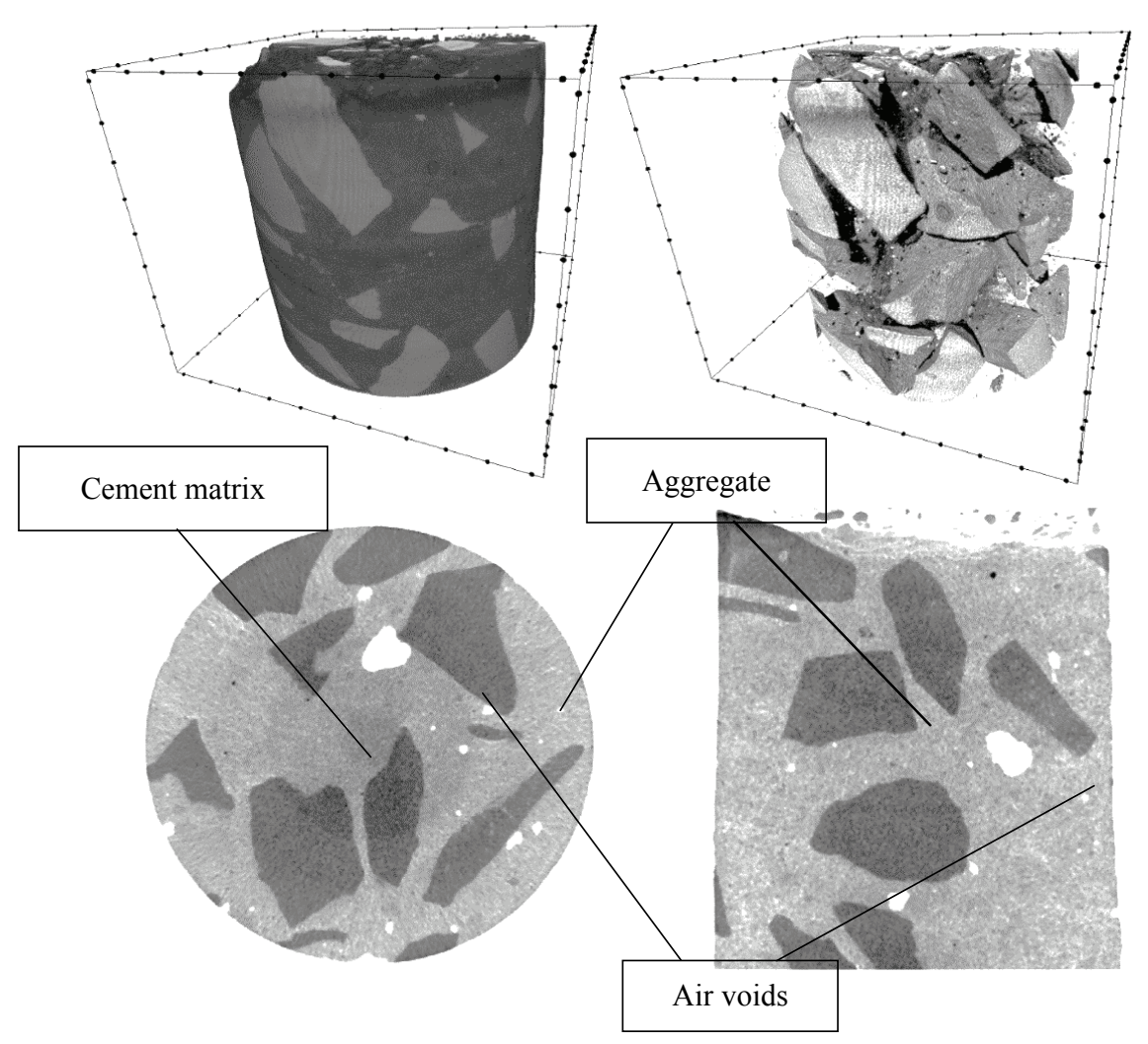

Fig. 3. Results of the imaging of the concrete sample

Table 1. Results of the 3D analysis of the sample

\begin{tabular}{|c|c|c|}
\hline Parameter name & $\begin{array}{c}\text { Parameter } \\
\text { symbol }\end{array}$ & Result \\
\hline Total VOI volume $\left[\mathrm{mm}^{3}\right]$ & $T V$ & 44421.5 \\
\hline Object volume $\left[\mathrm{mm}^{3}\right]$ & Obj.V & 42165.1 \\
\hline Percent object volume [\%] & Obj.V/TV & 94.9 \\
\hline Total VOI surface $\left[\mathrm{mm}^{2}\right]$ & $T S$ & 7283.5 \\
\hline Object surface $\left[\mathrm{mm}^{2}\right]$ & Obj.S & 15030.2 \\
\hline Intersection surface $\left[\mathrm{mm}^{2}\right]$ & i.S & 5848.2 \\
\hline Object surface/volume ratio $[1 / \mathrm{mm}]$ & Obj.S/Obj.V & 0.35 \\
\hline Object surface density $[1 / \mathrm{mm}]$ & Obj.S/TV & 0.33 \\
\hline Surface convexity index $[1 / \mathrm{mm}]$ & $S C v . I$ & -5.62 \\
\hline Centroid $(x)[\mathrm{mm}]$ & Crd.X & 25.51 \\
\hline Centroid $(y)[\mathrm{mm}]$ & Crd.Y & 26.13 \\
\hline Centroid $(z)[\mathrm{mm}]$ & $C r d . Z$ & 25.29 \\
\hline Structure thickness [mm] & St.Th & 5.04 \\
\hline Structure linear density $[1 / \mathrm{mm}]$ & St.Li.Dn & 0.19 \\
\hline Structure separation $[\mathrm{mm}]$ & $S t . S p$ & 2.42 \\
\hline Number of objects $[-]$ & Obj.N & 183 \\
\hline Number of closed pores [-] & $\operatorname{Po.N}(\mathrm{cl})$ & 23229 \\
\hline Volume of closed pores $\left[\mathrm{mm}^{3}\right]$ & Po.V(cl) & 281.1 \\
\hline Surface of closed pores $\left[\mathrm{mm}^{2}\right]$ & Po.S(cl) & 3649.5 \\
\hline Closed porosity (percent) [\%] & $P o(c l)$ & 0.66 \\
\hline Volume of open pore space $\left[\mathrm{mm}^{3}\right]$ & Po.V(op) & 1975.18 \\
\hline Open porosity (percent) [\%] & $P o(o p)$ & 4.45 \\
\hline Total volume of pore space $\left[\mathrm{mm}^{3}\right]$ & Po.V(tot) & 2256.3 \\
\hline Total porosity (percent) [\%] & $P o($ tot $)$ & 5.08 \\
\hline Euler number $[-]$ & Eu.N & 28393 \\
\hline Connectivity $[-]$ & Conn & -4981 \\
\hline Connectivity density $\left[1 / \mathrm{mm}^{3}\right]$ & Conn.Dn & -0.11 \\
\hline
\end{tabular}


composite are distinguishable based on the differences in the absorption of the X-ray radiation: cement matrix (light grey), aggregate (dark grey) and air voids (white). It can be noticed that the aggregate has significantly higher density range than the cement matrix.

The images of the material microstructure were subjected to various image processing techniques, e.g., filtering (median filter) in order to acquire proper segmentation of the components. Afterwards, the initial binarization of images was performed where the structure of the material was separated from the pores. Based on the binarized images, a 3D analysis was performed. The results of the aforementioned calculations are listed in Table 1.

For concrete, porosity is one of the most significant parameters that has influence on the mechanical properties of the material. Therefore, it was crucial to investigate the porosity profile along the height of the sample. The results of this analysis are shown in Fig. 4.
It is noteworthy that there is no evident trend of the porosity changes versus height of the sample. It is clear that, in general, the porosity is relatively low throughout the material with several peaks, indicating the presence of larger air voids.

The porosity analysis included also the calculation of pores distribution. It is observable that the distribution is uniform without any tendencies. On the contrary, the distribution of the aggregate has a significant peak around the value of $10 \mathrm{~mm}$. It indicates that the coarse aggregate comprises major part of the sample volume.

Another parameter calculated based on the images was the shape factor - sphericity. Sphericity is a measure of how spherical a 3D object is and it can be calculated according to the following formula

$$
S p h=\frac{\sqrt[3]{\pi}(6 V)^{2 / 3}}{S}
$$
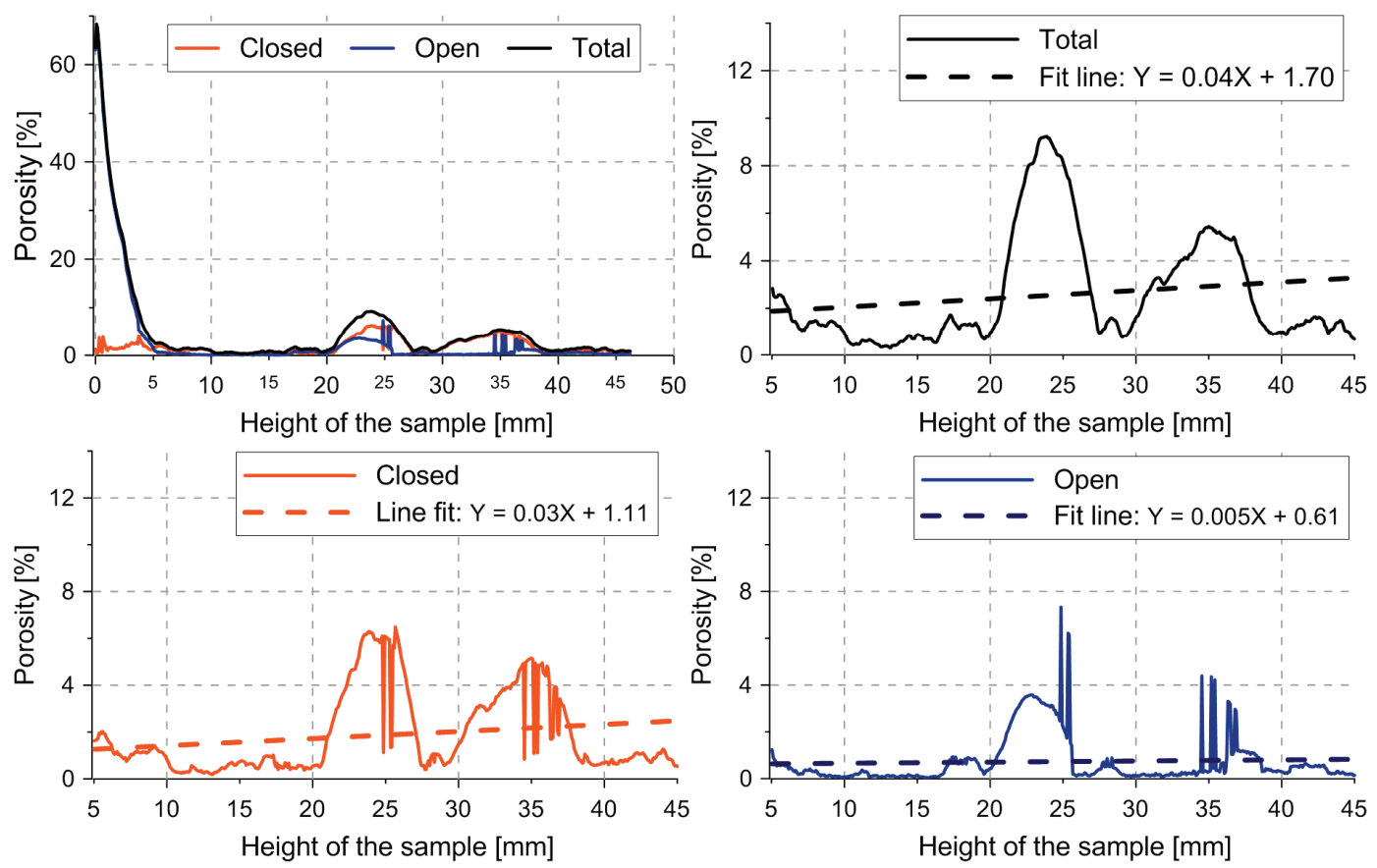

Fig. 4. Results of the porosity calculation along the height of the sample
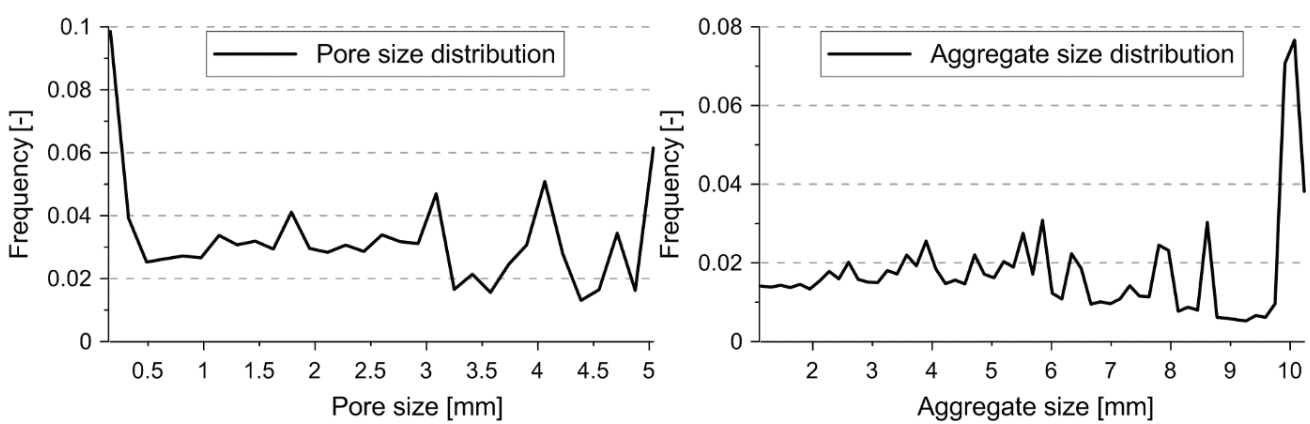

Fig. 5. Results of the pore and aggregate size distribution within the sample 
where $V$ and $S$ are the object volume and surface area, respectively.

For complex, non-spherical objects the surface area of the volume equivalent sphere will be much smaller than the particle surface area, thus Sph will be low. The maximum value possible is 1 , which would be obtained for a sphere. The results of the sphericity analysis for pores and aggregate are presented in Fig. 6. It is evident that the above mentioned sphericity distributions are significantly different. The majority of pores has sphericity value equal to $0.8-0.9$, depicting that their shape is similar to a sphere. On the other hand, the sphericity distribution of aggregate is comparable to the normal distribution with the mean value of around 0.6.
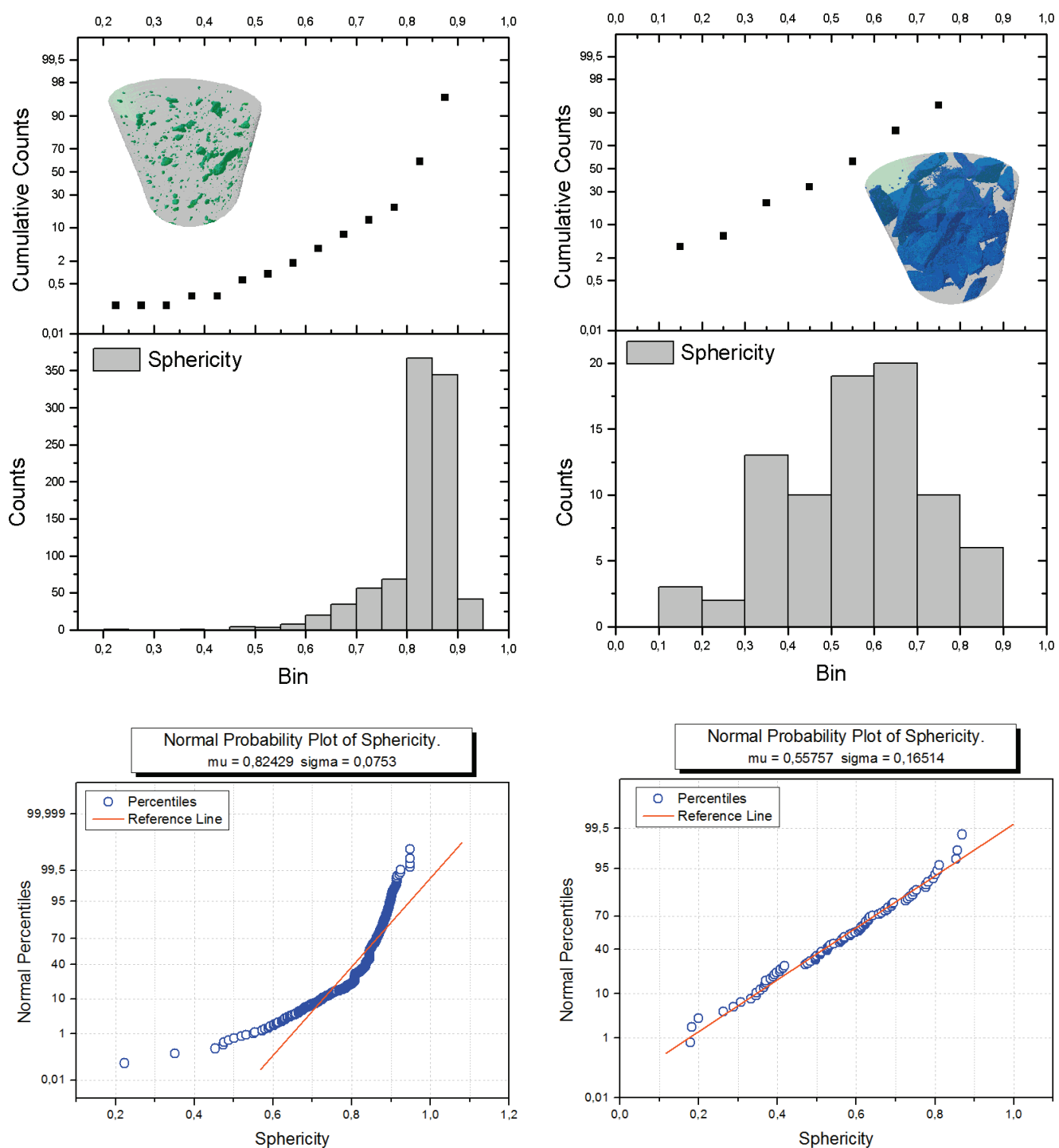

Fig. 6. Results of the sphericity calculation for pores and aggregate
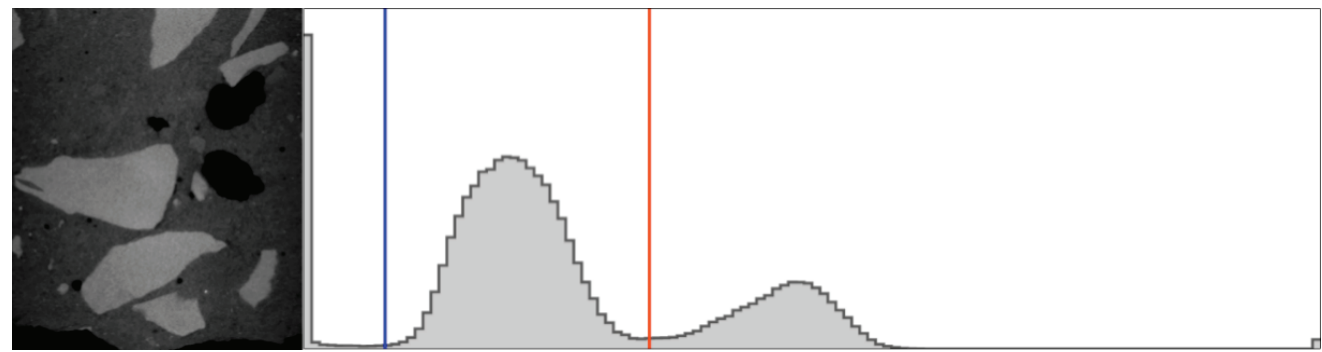

Fig. 7. Image and the corresponding histogram with selected thresholds: blue threshold - pores, red threshold - aggregate 
The basic measure to describe the microstructure of the material based on the $\mathrm{X}$-ray microCT images is the attenuation profile. As was mentioned in Section 2, according to equation (2), attenuation of the material is proportional to its density. Thus, the attenuation profiles can be interpreted as the density relations. However, the question arises: how to calculate the average value of the attenuation in order to get the proper value for the material? In this paper, two methods are presented in order to emphasise the differ-

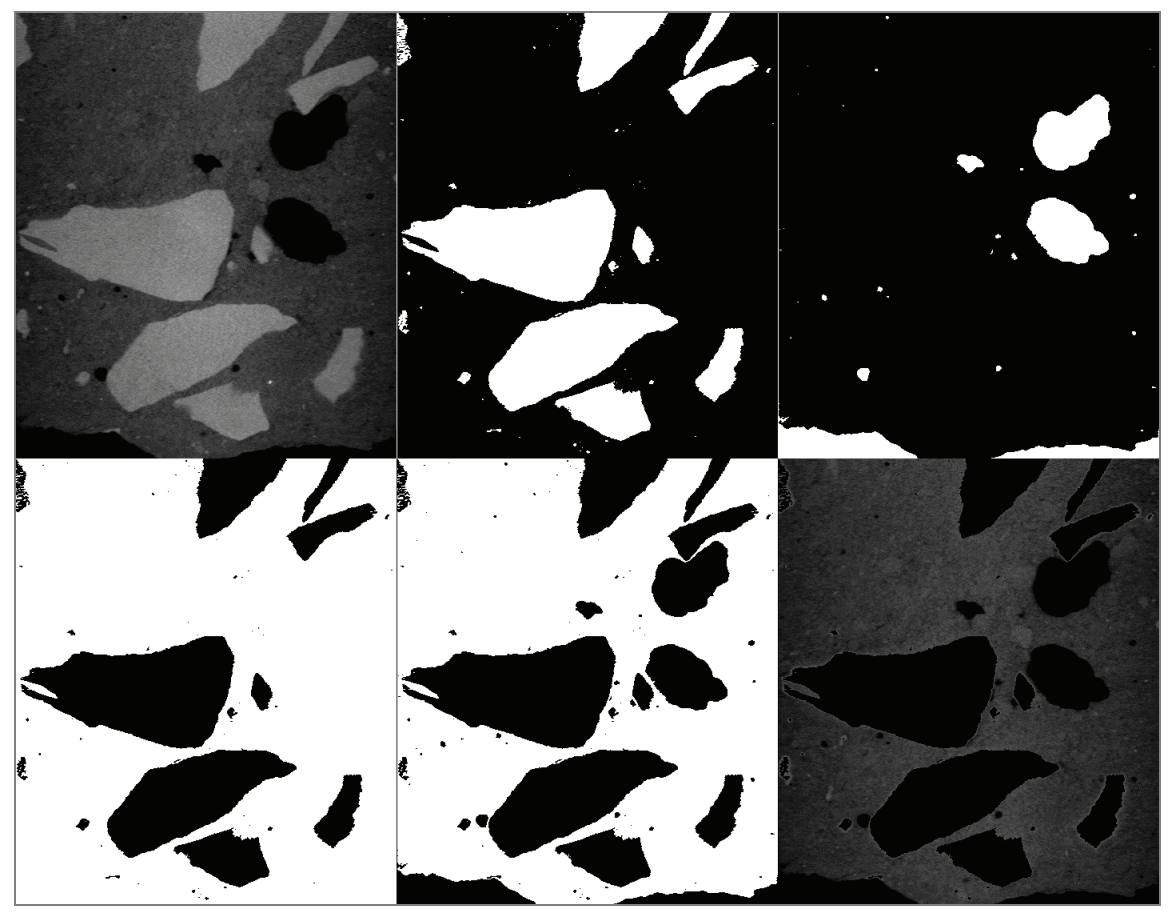

Fig. 8. Binary operations on the image: (a) original (raw) image, (b) aggregate segmentation, (c) air segmentation (pores), (d) subtraction of the images (a) and (b),

(e) subtraction of the images (d) and (c), (d) the resulting image of the cement matrix
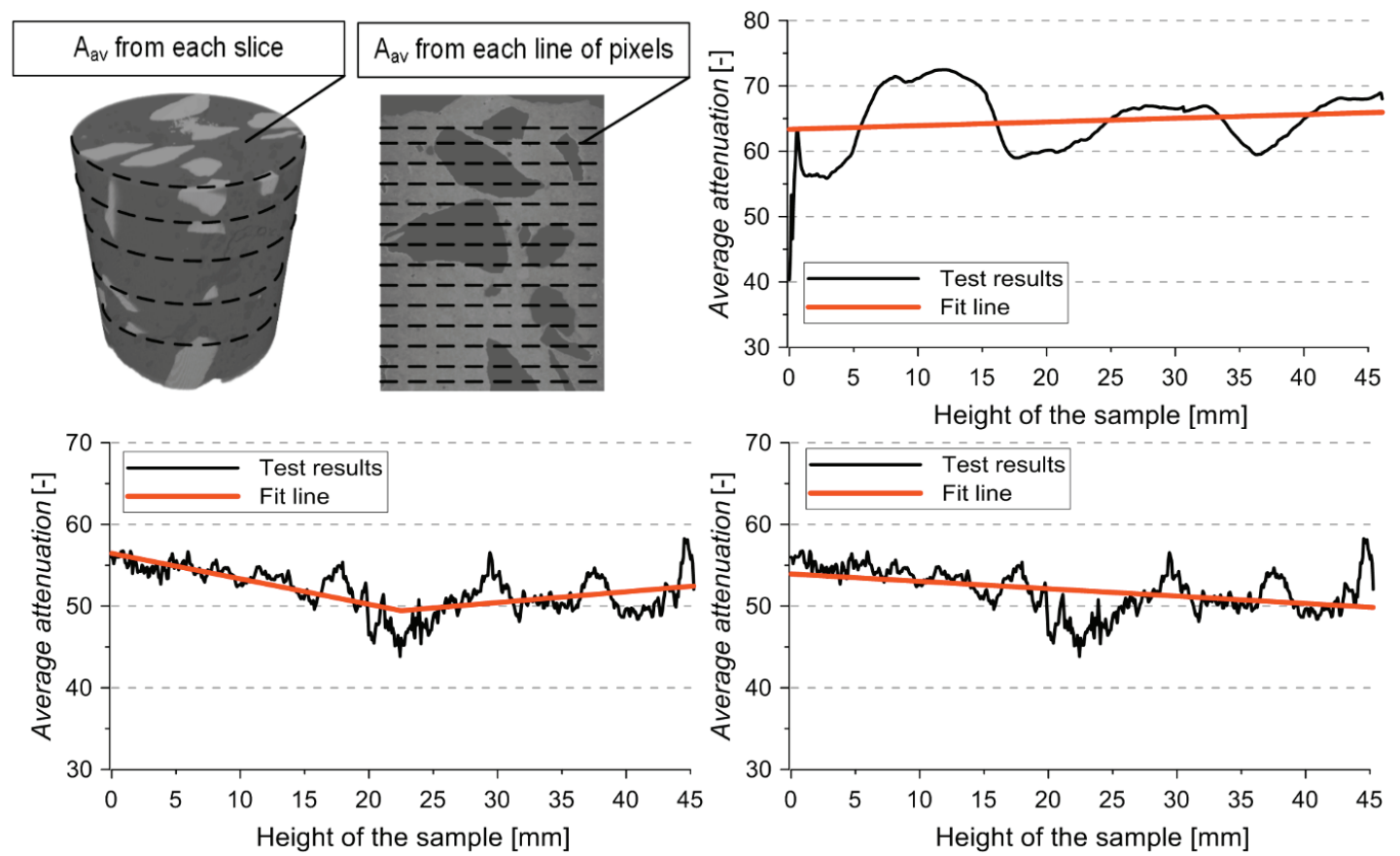

Fig. 9. Attenuation profile vs height of the sample: (a) two calculation methodologies,

(b) results of the calculation for each slice, (c) results of the calculation for each line of pixels - double curve fitting,

(d) results of the calculation for each line of pixels - single curve fitting 
ences in the results. In the first method, the attenuation was calculated as the average value of all the pixels for each "slice", whereas in the second one, an exemplary vertical cross-section was chosen and average attenuation was calculated as a mean value of all the pixels in each row of the image (Fig. 9a). It is important that in the first case no prior binary operations were performed on the images. On the contrary, in the second method, the images were preprocessed in order to obtain the pixel values of the matrix only - the aggregate and the pores were subtracted from the original image (Fig. 8). The thresholds for the image segmentation were chosen based on the image histogram and they are shown in Fig. 7.

The results of the attenuation profiles for both methods are shown in Fig. 9. It is clearly visible that the values calculated with the use of the first method
(Fig. 9b) are significantly different than the values obtained with the second method (Fig. 9c,d). In the first case, one can observe several peaks which increase the value of the average attenuation, indicating the presence of the aggregate on the set of slices. The second profile has a lower variance - the values are oscillating around one value. It is also noteworthy that the profiles have entirely different trend lines - in the first one the values are increasing whereas in the second one the trend is opposite.

After X-ray micro-CT investigation of the microstructure's geometry, the sample was tested in terms of its mechanical parameters by means of nanoindentation technique. For this purpose, a small fragment of the material was included in the epoxy resin with the use of vacuum machine in order to remove the air from the pores. Next, the surface of the sample was
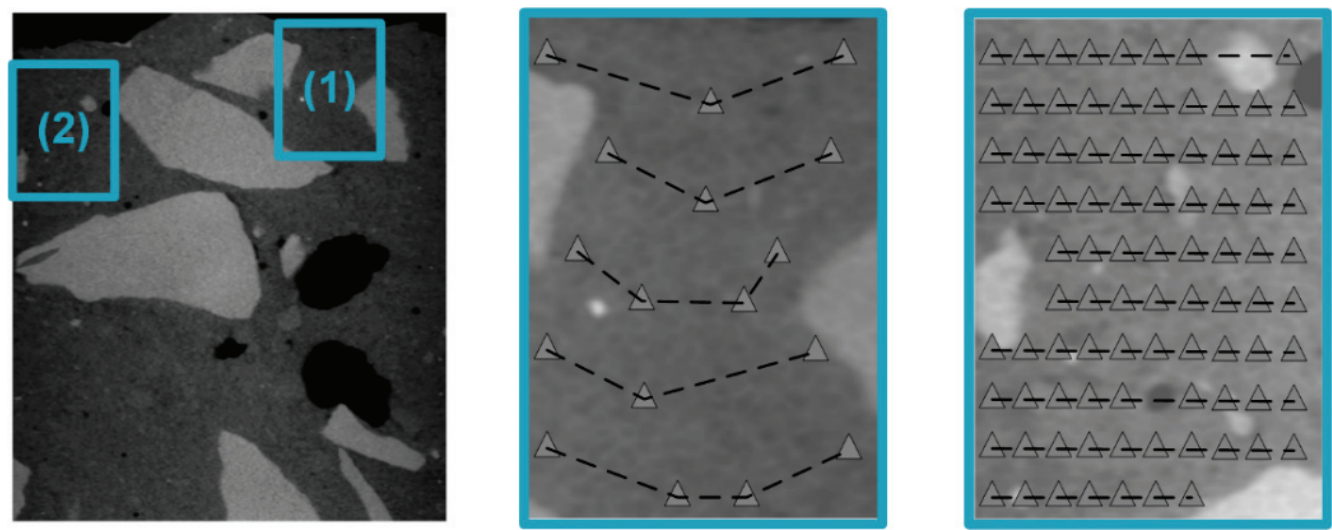

Fig. 10. Schemes of nanoindentation tests
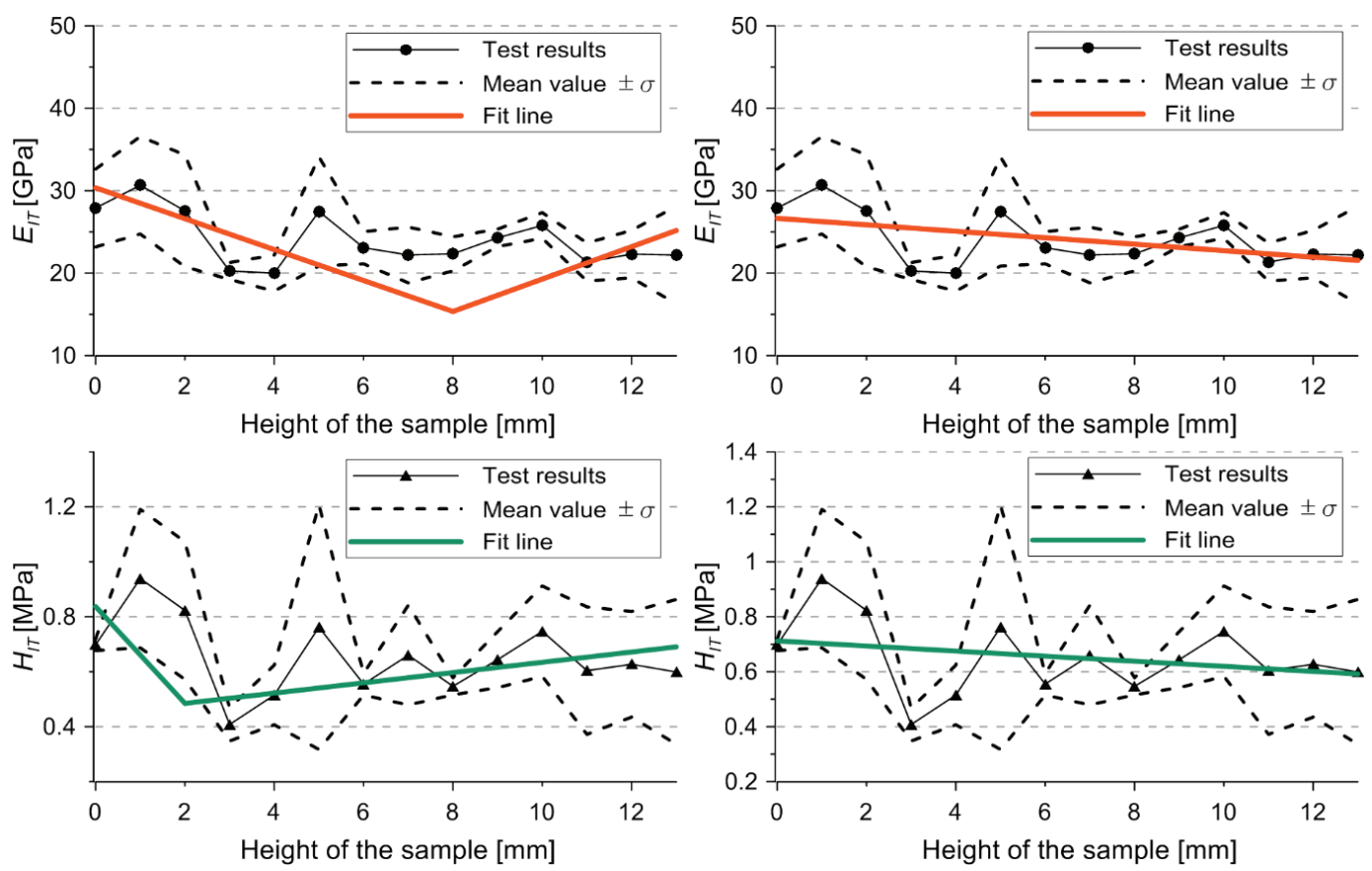

Fig. 11. Nanoindentation results $-3-4$ measurements 
polished to minimize the surface roughness. The measurements were conducted with the use of CSM Nanoindentation Tester (NHT). The value of the force used for loading of the sample was $500 \mathrm{mN}$. The methodology of the measurements is presented in Fig. 10. Firstly, the tests were conducted around every $1 \mathrm{~mm}$ and an average from 3-4 measurements taken in same area was calculated. Next, average from 10 measurements on each "line" was calculated and the tests were conducted every $0.2 \mu \mathrm{m}$. The locations for the nanoindentation were selected only in the cement matrix. Due to the fact that there were cases when the influence of the aggregate surrounding the measurement was noted, some of the measurements were eliminated.

The results of the average hardness HIT and Young's modulus EIT calculations are presented in Fig. 11 (for 3 measurements per average value) and in Fig. 12 (for 10 measurements per average value). It is clearly visible that the number of measurements has a significant influence on the results as well as on the trend line of the profile. The tendency has been investigated with the use of single and double fitting curves, in order to verify if there are any evident mechanical property changes with the height of the sample. It was notable that the trends differ depending on the number of measurements. One can observe that with the increasing number of tests per average value of the measured property, the trend line becomes almost uniform.

\section{CONCLUSIONS}

The paper presented two methods of microstructural analysis of materials, namely X-ray microcomputed tomography and nanoindentation. Both techniques are relatively new and have been used in material science for two decades. Even though they are becoming more and more popular among the scientists, there are still only several attempts to combine them in order to obtain full information about the object under investigation. The aim of the study was to prove the usefulness of such combination on a concrete sample. Concrete composite is one of the most popular construction materials in the world, thus, it was chosen as a potentially interesting and unusual material for this kind of modern measurements in micro scale. The article introduced the principles of the micro-CT testing as well as the background and theory of the nanoindentation technique. The results provided in the paper consisted of the material meas- ures of the microstructure geometry, e.g., porosity, pore size distribution, attenuation profiles, characterization of the aggregate shape and size distribution, sphericity of pores. Different methods of image processing and calculation were presented to show how crucial they are in terms of their influence on the final results of the aforementioned parameters. The geometrical study was followed by the nanoindentation measurements. The results of the hardness and Young's modulus were shown. Again, two different methods of analysis were presented to underline the importance of the adequate number of tests especially in the case of strongly heterogeneous material. The analysis showed that a probe which is not big enough or insufficient number of measurements may result in false and misleading outcome.

Summarizing, the methods presented proved to be useful when combined together. Even though each of them has broad possibilities, applied together, they give a complete and thorough data about material microstructure. Nevertheless, the study demonstrated that the statistical analysis of the acquired information plays a critical role in the process of the investigation. Furthermore, the preprocessing of data, e.g., image processing techniques in the case of microtomography are the major factor influencing the results. This is the reason why the aforementioned methods should be applied with understanding and caution. Undoubtedly, the future research is needed in this area in order to develop those methods and adjust them for specific materials.

\section{REFERENCES}

[1] Henry M., Darma I.S., Sugiyama T., Analysis of the effect of heating and re-curing on the microstructure of highstrength concrete using $X$-ray $C T$, Construction and Building Materials, 2014, Vol. 67, 37-46.

[2] Ren W., Yang Z., Sharma R., Zhang C., Withers P.J., Two-dimensional X-ray CT image based meso-scale fracture modelling of concrete, Engineering Fracture Mechanics, 2015, Vol. 133, 24-39.

[3] Bossa N., Chaurand P., Vicente J., Borschneck D., Levard C., Aguerre-Chariol O., Rose J., Micro- and nano$X$-ray computed-tomography: A step forward in the characterization of the pore network of a leached cement paste, Cement and Concrete Research, 2015, Vol. 67, 138-147.

[4] Korata L., Ducmana V., Legata A., Mirtic B., Characterization of the pore-forming process in lightweight aggregate based on silica sludge by means of X-ray microtomography (micro-CT) and mercury intrusion porosimetry (MIP), Ceramics International, 2013, Vol. 39, 6997-7005.

[5] Munkholm L.J., Heck R.J., Deen B., Soil pore characteristics assessed from $X$-ray micro-CT derived images and correlations to soil friability, Geoderma, 2012, Vol. 181-182, 22-29. 
[6] Liu T., Zhang X., Li Z., Chen Z., Research on the homogeneity of asphalt pavement quality using $X$-ray computed tomography (CT) and fractal theory, Construction and Building Materials, 2014, Vol. 68, 587-598.

[7] Steppe K., CNudde V., Girard C., Lemeur R., Cnudde J.P., JACOBS P., Use of X-ray computed microtomography for noninvasive determination of wood anatomical characteristics, Journal of Structural Biology, 2004, Vol. 148, 11-21.

[8] Constantinides G., Ravi Chandran K.S., Ulm F.-J., VAN Vliet K.J., Grid indentation analysis of composite microstructure and mechanics: Principles and validation, Materials Science and Engineering, 2006, Vol. 430, 189-202.

[9] Sorelli L., Constantinides G., Ulm F.-J., Toutlemonde F., The nano-mechanical signature of Ultra High Performance Concrete by statistical nanoindentation techniques, Cement and Concrete Research, 2008, Vol. 38, 1447-1456.

[10] Zhu W., Hughes J.J., Bicanic N., Pearce C.J., Nanoindentation mapping of mechanical properties of cement paste and natural rocks, Materials Characterization, 2007, Vol. 58, 1189-1198.
[11] ZADPOOR A.A., Nanomechanical characterization of heterogeneous and hierarchical biomaterials and tissues using nanoindentation: The role of finite mixture models, Materials Science and Engineering C, 2015, Vol. 48, 150-157.

[12] Díez-Pascual A.M., Gómez-Fatou M.A., ANia F., Flores A., Nanoindentation in polymer nanocomposites, Progress in Materials Science, 2015, Vol. 67, 1-94.

[13] Sтоск S.R., MicroComputed tomography: methodology and applications, Boca Raton: CRC Press, 2009.

[14] EPSTEIN C.L., Introduction to mathematics of medical imaging, SIAM, Philadelphia, 2003.

[15] DOERNER M.F., NIX W.D., A method for interpreting the data from depth-sensing indentation instruments, Journal of Material Research, 1986, Vol. 1(4), 601-609.

[16] Oliver W.C., Pharr G.M., An improved technique for determining hardness and elastic modulus using load and displacement sensing indentation experiments, Journal of Material Research, 1992, Vol. 7(6), 1564-1583.

[17] FISCHER-CRIPPS A., Nanoindentation, Springer, New York, 2011. 